\title{
Central extracorporeal membrane oxygenation and early rehabilitation for persistent severe pulmonary hypertension following pulmonary endarterectomy
}

\author{
Gil Myeong Seong ${ }^{1}$, Sang-Bum Hong ${ }^{1}$, Jin Won Huh ${ }^{1}$, Chae-Man Lim ${ }^{1}$, Younsuck Koh ${ }^{1}$, Jae Won Lee ${ }^{2,3}$, \\ Sung-Ho Jung ${ }^{2}$, Duck-Woo Park ${ }^{3,4}$, Jae Seung Lee ${ }^{1,3}$ \\ Departments of ${ }^{1}$ Pulmonary and Critical Care Medicine and ${ }^{2}$ Thoracic and Cardiovascular Surgery, ${ }^{3}$ Center for Pulmonary Hypertension and Venous \\ Thrombosis, and ${ }^{4}$ Department of Cardiology, Asan Medical Center, University of Ulsan College of Medicine, Seoul, Korea
}

Chronic thromboembolic pulmonary hypertension is potentially curable with a pulmonary endarterectomy. However, approximately 20\% of patients have persistent pulmonary hypertension after pulmonary endarterectomy, which is a major risk factor for postoperative death. Here, we report a 34-year-old woman who suffered persistent severe pulmonary hypertension following a successful pulmonary endarterectomy for chronic thromboembolic pulmonary hypertension. Extracorporeal membrane oxygenation (ECMO) and atrial septostomy were successfully performed as rescue treatments, and active rehabilitation during ECMO was prescribed to facilitate recovery.

Key Words: endarterectomy; extracorporeal membrane oxygenation; pulmonary hypertension; rehabilitation

Pulmonary endarterectomy (PEA) is the current treatment of choice for chronic thromboembolic pulmonary hypertension (CTEPH) [1]. A successful operation markedly improves patient symptoms, and functional status and the hemodynamics of the pulmonary vasculature and right ventricle [2]. Despite the favorable long-term prognosis of PEA, there are specific perioperative complications, such as persistent pulmonary hypertension $(\mathrm{PH})$ and reperfusion lung injury, that account for the majority of deaths [3,4]. Moreover, some patients experience a pulmonary hypertensive crisis immediately following endarterectomy, making it very difficult to wean them off cardiopulmonary bypass (CPB). For such complex cases, venoarterial extracorporeal membrane oxygenation (VA-ECMO) support is the only effective treatment option for recovery. We present a difficult case of CTEPH with antiphospholipid syndrome wherein VA-ECMO and atrial septostomy were used as salvage therapies during early PEA failure. This case demonstrates the life-threatening nature of postPEA complications and provides an effective solution.

\section{CASE REPORT}

A 34-year-old woman was referred to Asan Medical Center for pulmonary hypertension for further evaluation. Approximately 15 years prior, she had been diagnosed with pulmonary

\section{Case Report}

Received: December 29, 2016 Revised: September 22, 2017

Accepted: September 26, 2017

Corresponding author Jae Seung Lee

Department of Pulmonary and Critical Care Medicine, Asan Medical Center, University of Ulsan College of Medicine, 88 Olympic-ro 43-gil, Songpa-gu, Seoul 05505, Korea Tel: +82-2-3010-3394

Fax: +82-2-2045-4039 E-mail: jsdoc1186@daum.net

Copyright () 2019 The Korean Society of Critical Care Medicine

This is an Open Access article distributed under the terms of Creative Attributions Non-Commercial License (http:// creativecommons.org/li-censes/by-nc/4.0/) which permits unrestricted noncommercial use, distribution, and reproduction in any medium, provided the original work is properly cited. 
thromboembolism, systemic lupus erythematosus, and antiphospholipid syndrome. Despite anticoagulant treatment with warfarin, she developed CTEPH and was placed on the waitlist for a heartlung transplantation, which she was on for 2 years. Two months before admission, she was treated for community-acquired pneumonia. After recovering, she gained 5 kg and presented with World Health Organization functional class IV symptoms. She had no active systemic lupus erythematosus-related symptoms but did have a history of hypothyroidism. Her medications included sildenafil, bosentan, furosemide, spironolactone, warfarin, digoxin, hydroxychloroquine, and levothyroxine. On examination, her vitals were as follows: blood pressure, 104/70 mmHg; pulse, 99 beats/min; temperature, $36.9^{\circ} \mathrm{C}$; respiratory rate, 22 breaths $/ \mathrm{min}$; and oxygen saturation while breathing ambient air, $83 \%$.

The majority of routine laboratory test results were within the normal range, except for the following: prothrombin time, 1.68 international normalized ratio (INR; reference range, 0.8 to $1.3 \mathrm{INR}$ ); brain natriuretic peptide level, $564 \mathrm{pg} / \mathrm{mL}$ (reference range, 0 to $100 \mathrm{pg} / \mathrm{mL}$ ); and $\mathrm{N}$-terminal pro-brain natriuretic peptide level, 4,108 pg/mL (reference range, 0 to $100 \mathrm{pg} / \mathrm{mL}$ ). Chest radiography revealed severe cardiomegaly and enlarged pulmonary arteries (Figure 1). Chest computed tomography revealed a severely enlarged pulmonary trunk and central pulmonary arteries with multiple wall calcifications and large organized thrombi in the right interlobar, right lower lobar, and left upper lobar branches (Figure 2A). Lung ventilation/perfusion scan showed multiple mismatched perfusion defects in both lobes (Figure 2B). Transthoracic echocardiography revealed a severe right ventricular dysfunction with a thickened ventricular free wall and peak tricuspid regurgitant velocity of $5.0 \mathrm{~m} / \mathrm{s}$. Her 6-minute walk distance was $220 \mathrm{~m}$. Right heart catheterization (RHC) with pulmonary angiography showed a mean pulmonary arterial pressure (PAP) of $78 \mathrm{mmHg}$ (96/65), cardiac index of $2.27 \mathrm{~L} / \mathrm{min} / \mathrm{m}^{2}$, pulmonary artery wedge pressure of $5 \mathrm{mmHg}$, pulmonary vascular resistance of $25.97 \mathrm{wood}$ units, and mean right atrial pressure of $13 \mathrm{mmHg}$, which were confirmatory for CTEPH.

Thus, the patient was diagnosed as having very severe $\mathrm{PH}$ with advanced right ventricular failure and worsening symptoms. The Pulmonary Hypertension Team comprising a pulmonologist, cardiologist, and thoracic surgeon determined the patient to be eligible for PEA before lung transplantation because lung transplantation is the only potential treatment option for patients with CTEPH who are either inoperable or who experienced treatment failure with PEA. Furthermore, donor availability is generally limited, especially for heart-lung trans-

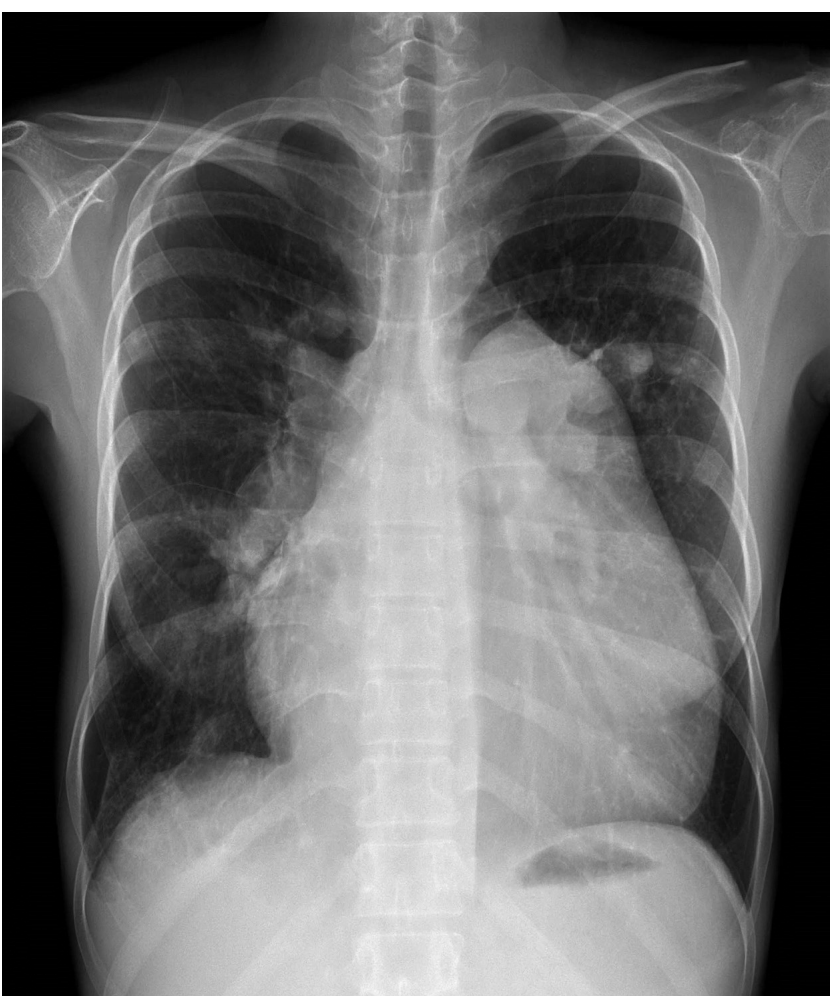

Figure 1. Preoperative chest radiograph shows enlargement of the main pulmonary artery and of the right and left pulmonary arteries with peripheral pruning. The heart is enlarged with a prominent right atrial contour.

plantation.

Bilateral PEA was performed with the patient under deep hypothermia $\left(25^{\circ} \mathrm{C}\right)$ and circulatory arrest [5]. After median sternotomy, the ascending aorta and both vena cava were cannulated, and CPB was initiated. The patient was cooled, and a left atrial vent was placed through the right upper pulmonary vein. The thickened intima and old organized thrombi were removed from both the lobar and segmental arteries via main and right pulmonary artery incisions (Figure 3 ). The total duration of circulatory arrest was 29 minutes. After being weaned off $\mathrm{CPB}$, the patient was hemodynamically unstable and hypoxemic. Despite an apparently successful endarterectomy, systolic PAP was suprasystemic at $100 \mathrm{mmHg}$. CPB was resumed, and an atrial septal defect was created to decompress the right heart; however, the weaning trials failed again. Consequently, $14 \mathrm{~F}$ arterial and $24 \mathrm{~F}$ venous catheters were inserted into the right femoral vessels, and VA-ECMO was initiated using a Rotaflow centrifugal pump with a Quadrox-D oxygenator (MAQUET Cardiopulmonary AG, Hirrlingen, Germany). Afterward, the patient was transferred to the intensive care unit. Vasoactive drugs (i.e., norepinephrine, epinephrine, 


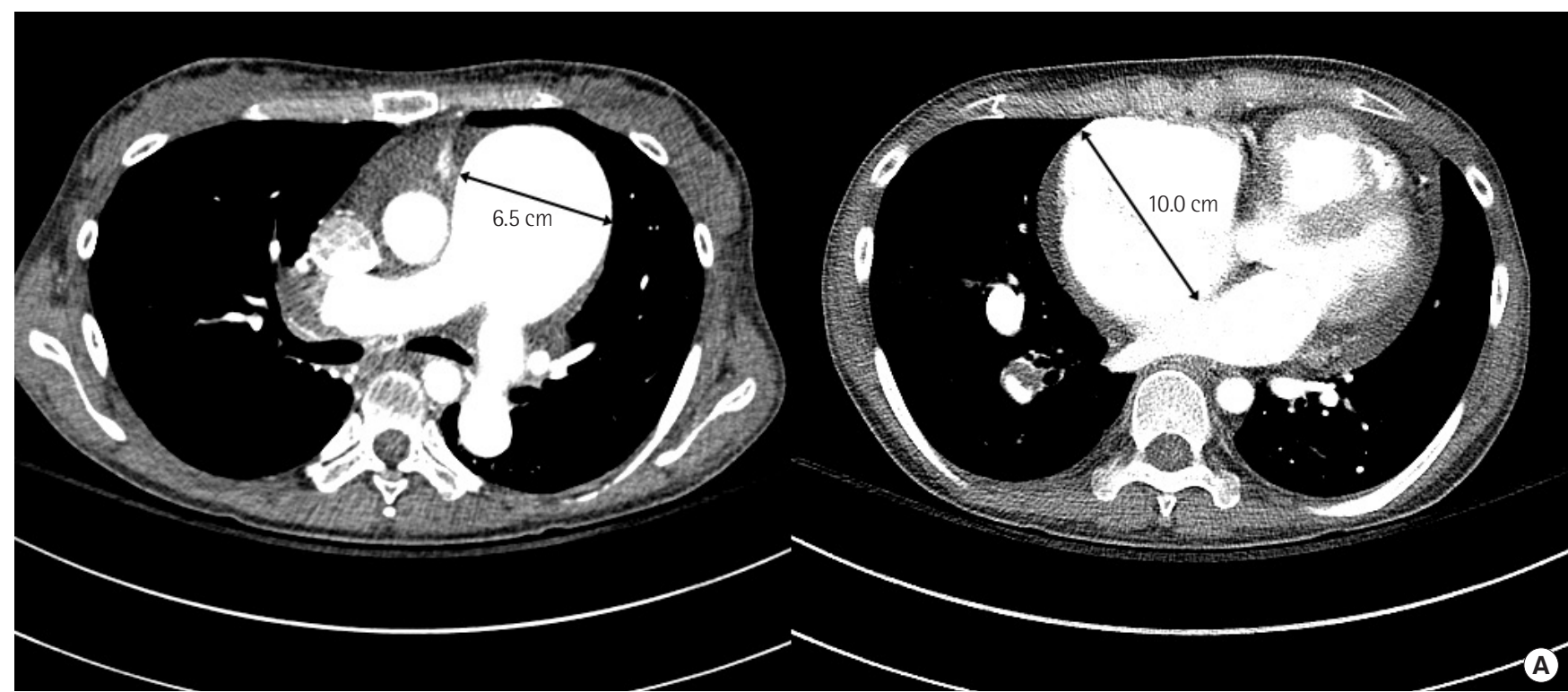

Lung perfusion scan 99mTc-MAA

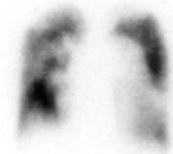

ANT

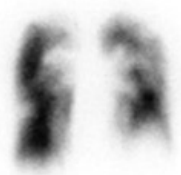

POST

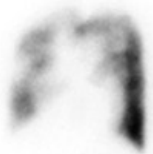

LAO

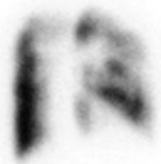

RPO

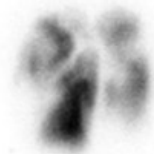

LPO

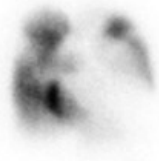

RAO

Lung inhalation scan technegas

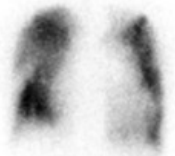

ANT

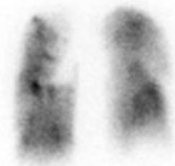

POST

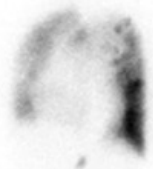

LAO

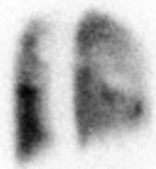

RPO

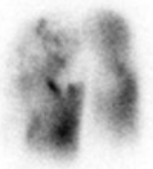

LPO

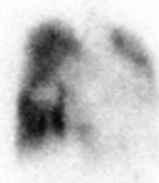

RAO

Figure 2. (A) Axial contrast-enhanced chest computed tomography confirms enlargement of the main pulmonary artery (6.5 $\mathrm{cm})$ and shows wall-adherent chronic thrombus with calcification in the right pulmonary artery. The image shows the dilated right atrium (10.0 $\mathrm{cm})$ and right ventricular hypertrophy. (B) Lung ventilation/perfusion scan reveals multiple mismatched perfusion defects in both lobes. MAA: macro aggregated albumin; ANT: anterior; LAO: left anterior oblique; LPO: left posterior oblique; POST: posterior; RPO: right posterior oblique; RAO: right anterior oblique.

and milrinone) and inhaled nitric oxide were administered to improve her hemodynamic status.

In the intensive care unit, intravenous unfractionated heparin was initiated 4 hours after surgery, and a negative fluid balance was maintained. Although it was difficult to wean the patient off ECMO support, a second RHC was performed on postoperative day (POD) 6. Pulmonary angiography revealed extensive reobstructions of both pulmonary arteries by acute thrombi (Figure 4), and her mean PAP remained extremely high (73 mmHg). Thus, we prepared other long-term treatment options, including lung transplantation and the following: (1) ECMO mode was changed to the central type using the right atrium and ascending aorta for early mobilization and active rehabilitation; (2) we attempted to use a relatively high heparin dose to maintain an activated partial thromboplastin time of 70-90 seconds and an anti-factor Xa $>0.3 \mathrm{IU} / \mathrm{ml}$ (therapeutic range, 0.3 to $0.7 \mathrm{IU} / \mathrm{ml}$ ) because of recurrent thrombosis in the pulmonary arteries despite immediate anticoagulation therapy; and (3) medical management of residual PH was initiated with sildenafil and bosentan while maintaining a negative fluid balance.

On POD 6, the patient was cannulated after redo-sternotomy for central ECMO using a 20F-angled arterial cannula in the ascending aorta and a $28 \mathrm{~F}$-angled venous cannula in the right atrium. The sternum was closed, and cannulas were tunneled in the subxiphoid position (Figure 5). 


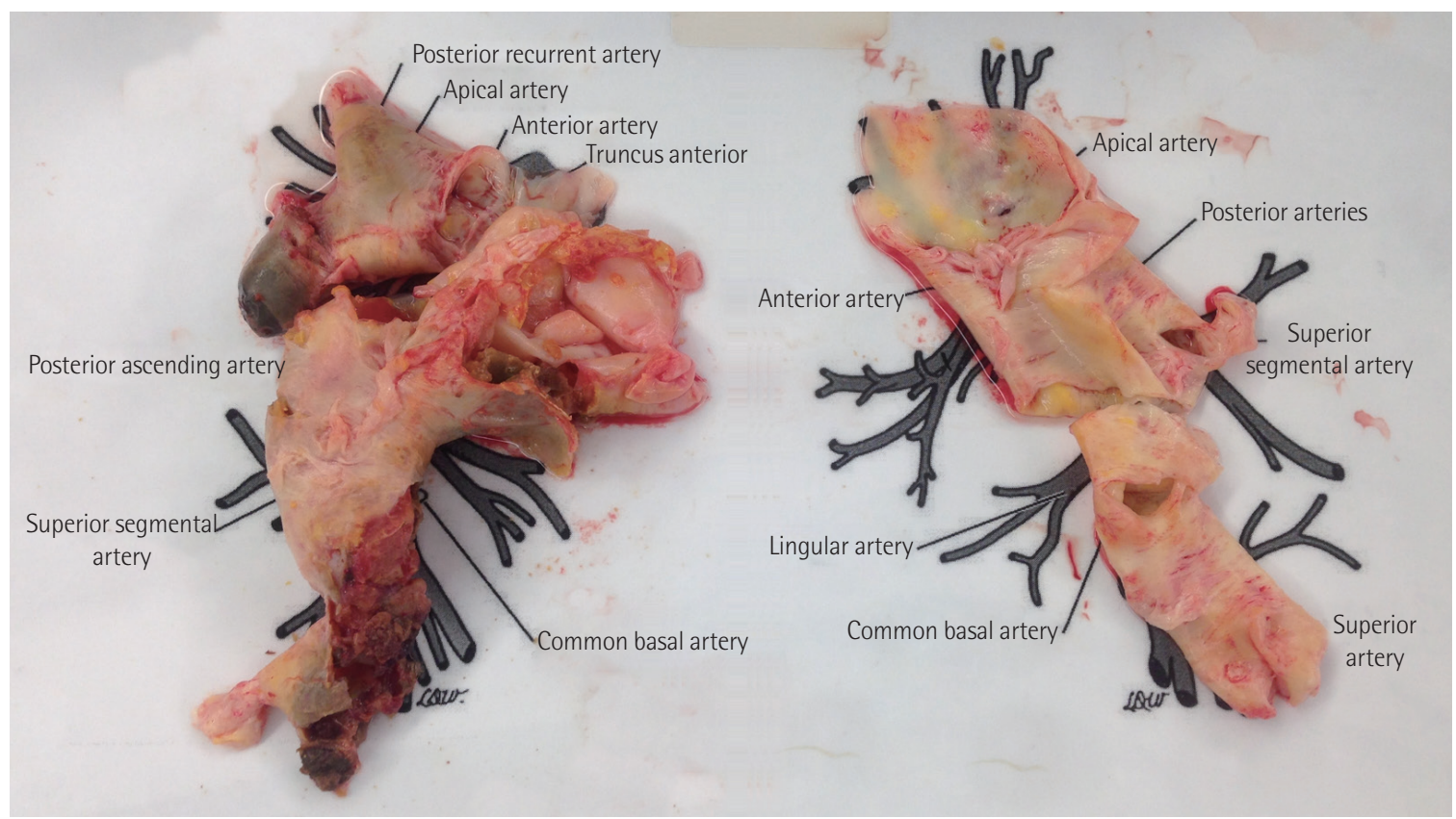

Figure 3. Specimens removed from the pulmonary arteries during pulmonary endarterectomy.
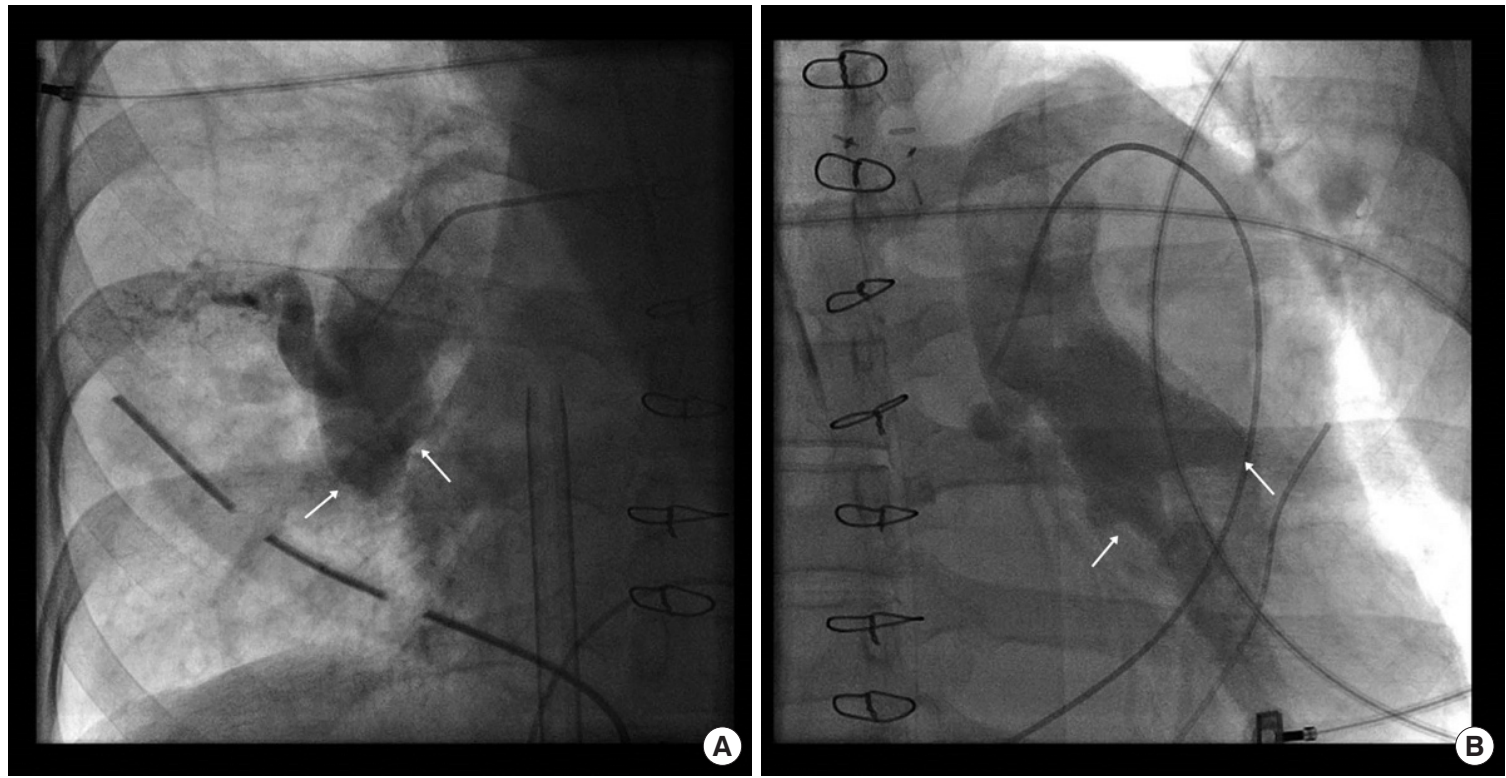

Figure 4. Postoperative pulmonary angiography reveals total occlusion (arrows) of the right (A) and left (B) basal trunks of the pulmonary artery.

Active rehabilitation was performed 2-3 times/day by physical therapists, intensive care physicians, respiratory therapists, or nurses. One day after central ECMO support was performed (POD 7); the patient was able to independently sit on the edge of the bed with ECMO and mechanical ventilator support; and eventually she was able to stand without support and march in place. After these improvements, all vasoactive drugs were discontinued, and extubation was success- fully performed on POD 11. After extubation, she was able to ambulate independently with only ECMO support.

On POD 14, she was hemodynamically stable, with only $20 \%$ of cardiac index support performed by ECMO. Follow-up echocardiography revealed a somewhat decreased right ventricle size and peak tricuspid regurgitant velocity. At this point, the Pulmonary Hypertension Team decided to discontinue ECMO support and medically treat the patient rather than 


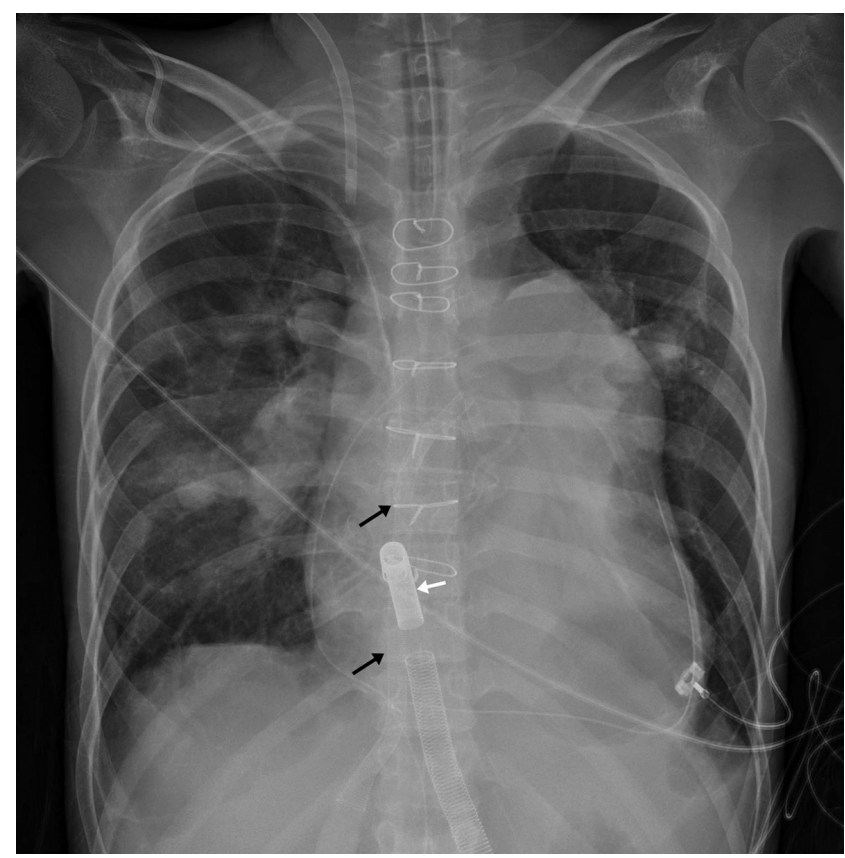

Figure 5. Chest radiograph obtained after central-type conversion shows the drainage cannula in the right atrium (white arrow) and the return cannula in the ascending aorta (black arrows).

perform lung transplantation. The central ECMO support was eventually discontinued under general anesthesia with intubation on POD 14, and she was successfully weaned off the mechanical ventilator on POD 22. Subsequently, the patient was transferred to the general ward, and oral anticoagulant and diuretic doses were carefully titrated. On POD 43 , she was finally discharged with a prescription of long-term oxygen therapy and a plan to undergo balloon pulmonary angioplasty.

Approximately 3 months after her operation, the patient was deemed to be World Health Organization functional class II. Her follow-up RHC revealed total occlusion of the right upper trunk and both multiple segmental arteries; she had a mean PAP of $50 \mathrm{mmHg}$ and pulmonary vascular resistance of 9.5 wood units. The patient can tolerate her current medical treatments, and additional intervention will be determined during regular follow-up.

Written informed consent was obtained from the patient for publication of this case report and accompanying images.

\section{DISCUSSION}

Herein, we report a case of severe residual $\mathrm{PH}$ following successful PEA. In CTEPH cases, pulmonary hypertension results from both persistent macrovascular obstruction and smallvessel arteriopathy and neurohumoral factors that mediate vasoconstriction. According to a recent prospective cohort study [4], postoperative residual PH was observed in 15\%-18\% of patients and was associated with increased mortality (hazard ratio, 3.66; $\mathrm{P}=0.0008$ ). However, neither the incidence nor the outcome of early PEA failure has been reported to date.

For these difficult situations, postoperative ECMO is essential and recommended as a standard of care in pulmonary hypertension centers. According to previous retrospective studies $[6,7]$, approximately $4 \%-20 \%$ of patients required postoperative extracorporeal life support. Atrial septostomy was traditionally used as an effective palliative treatment for right heart failure in severe $\mathrm{PH}$ cases. Although many effective targeted therapies for $\mathrm{PH}$ have since been developed, atrial septostomy still plays a partial role for immediate [8] and long-term outcomes [9].

Our patient had CTEPH with autoimmune thrombophilia. Following early failure of PEA, salvage atrial septostomy failed to wean the patient off $\mathrm{CPB}$, and VAECMO was necessary. Moreover, follow-up RHC revealed recurrent extensive thrombosis in both pulmonary arteries. In this situation, the two major treatment options were lung transplantation or medical treatment, including balloon pulmonary angioplasty. Lung transplantation was considered the inferior option for the following reasons: (1) the patient was young, and the 5-year survival rate is only approximately $50 \%$ following transplantation [10], which, in her case, would be worse considering her thrombogenic and postoperative condition, which requires extracorporeal life support [7,11,12]; (2) she was returning to a good performance state through early rehabilitation with central-type ECMO support; and (3) there would be additional opportunities to modify her medication or perform balloon angioplasty after resolving acute thrombi. Sequential pulmonary balloon angioplasty for distal arteries has been recently used for selected inoperable CTEPH patients or patients with residual or recurrent $\mathrm{PH}$ after successful PEA [13-15].

Rehabilitation during VA-ECMO is challenging. Most techniques rely on femoral cannulation, thereby immobilizing patients, which in turn can lead to rapid deconditioning [16]. ECMO using the aorta or upper-extremity arteries can liberate both legs and allow the patient to undergo active physical therapy. Early mobilization is beneficial for selected patients receiving ECMO who are waiting for recovery or lung transplantation. At our center, ECMO patients are evaluated daily to assess hemodynamic and respiratory stability and suitability for rehabilitation and mobilization. The exercise protocol begins with sitting on the edge of the bed and progresses to exercises performed in the standing position; the ultimate goal is for the patient to be able to march and ambulate safely. 
To achieve this, the multidisciplinary team pays attention to intravenous lines, ECMO cannulas, and monitoring devices currently in place. Maintenance of adequate oxygenation and hemodynamic stability during rehabilitation, ECMO sweep gas and blood flow rates, and supplemental oxygen can all be increased as needed.

In conclusion, PEA is a curative treatment option for CTEPH but can have life-threatening postoperative complications. ECMO is an essential treatment option in this situation, and early active rehabilitation during ECMO can facilitate recovery. Considering a multidisciplinary approach for complicated patients both preoperatively and postoperatively is important.

\section{CONFLICT OF INTEREST}

No potential conflict of interest relevant to this article was reported.

\section{ORCID}

Gil Myeong Seong https://orcid.org/0000-0002-0765-8273

Sang-Bum Hong ～https://orcid.org/0000-0003-2737-7695

Jin Won Huh https://orcid.org/0000-0002-3449-0461

Chae-Man Lim https://orcid.org/0000-0001-5400-6588

Younsuck Koh https://orcid.org/0000-0001-5066-2027

Jae Won Lee https://orcid.org/0000-0003-0751-2458

Sung-Ho Jung https://orcid.org/0000-0002-3699-0312

Duck-Woo Park https://orcid.org/0000-0001-6643-0239

Jae Seung Lee

\section{AUTHOR CONTRIBUTIONS}

Conceptualization: GMS, JSL. Data curation: GMS. Formal analysis: GMS, SBH, JSL. Visualization: GMS. Writing - original draft: GMS, JSL. Writing - review \& editing: all authors.

\section{REFERENCES}

1. Galiè N, Humbert M, Vachiery JL, Gibbs S, Lang I, Torbicki A, et al. 2015 ESC/ERS guidelines for the diagnosis and treatment of pulmonary hypertension: the Joint Task Force for the Diagnosis and Treatment of Pulmonary Hypertension of the European Society of Cardiology (ESC) and the European Respiratory Society (ERS). Eur Respir J 2015;46:903-75.

2. Madani MM, Auger WR, Pretorius V, Sakakibara N, Kerr KM, Kim NH, et al. Pulmonary endarterectomy: recent changes in a single institution's experience of more than 2,700 patients.

Ann Thorac Surg 2012;94:97-103.

3. Cannon JE, Su L, Kiely DG, Page K, Toshner M, Swietlik E, et al. Dynamic risk stratification of patient long-term outcome after pulmonary endarterectomy: results from the United Kingdom National Cohort. Circulation 2016;133:1761-71.

4. Delcroix M, Lang I, Pepke-Zaba J, Jansa P, D’Armini AM, Snijder R, et al. Long-term outcome of patients with chronic thromboembolic pulmonary hypertension: results from an international prospective registry. Circulation 2016;133:859-71.

5. Jamieson SW, Kapelanski DP, Sakakibara N, Manecke GR, Thistlethwaite PA, Kerr KM, et al. Pulmonary endarterectomy: experience and lessons learned in 1,500 cases. Ann Thorac Surg 2003;76:1457-62.

6. Boulate D, Mercier O, Mussot S, Fabre D, Stephan F, Haddad F, et al. Extracorporeal life support after pulmonary endarterectomy as a bridge to recovery or transplantation: lessons from 31 consecutive patients. Ann Thorac Surg 2016;102:260-8.

7. Nierlich P, Ristl R. Perioperative extracorporeal membrane oxygenation bridging in patients undergoing pulmonary endarterectomy. Interact Cardiovasc Thorac Surg 2016;22:181-7.

8. Barst RJ. Role of atrial septostomy in the treatment of pulmonary vascular disease. Thorax 2000;55:95-6.

9. Sandoval J, Gaspar J, Peña H, Santos LE, Córdova J, del Valle $\mathrm{K}$, et al. Effect of atrial septostomy on the survival of patients with severe pulmonary arterial hypertension. Eur Respir J 2011;38:1343-8.

10. Yusen RD, Christie JD, Edwards LB, Kucheryavaya AY, Benden C, Dipchand AI, et al. The registry of the International Society for Heart and Lung Transplantation: thirtieth adult lung and heart-lung transplant report--2013; focus theme: age. J Heart Lung Transplant 2013;32:965-78.

11. Valentine VG, Lombard GA. Connective tissue disorders. In: Vigneswaran WT, Garrity ER Jr, editors. Lung transplantation. 1st ed. Colchester: Informa Healthcare; 2010. p. 75-82.

12. Mason DP, Thuita L, Nowicki ER, Murthy SC, Pettersson GB, Blackstone EH. Should lung transplantation be performed for patients on mechanical respiratory support? The US experience. J Thorac Cardiovasc Surg 2010;139:765-73.e1.

13. Collaud S, Brenot P, Mercier O, Fadel E. Rescue balloon pulmonary angioplasty for early failure of pulmonary endarterectomy: the earlier the better? Int J Cardiol 2016;222:39-40.

14. Mizoguchi H, Ogawa A, Munemasa M, Mikouchi $\mathrm{H}$, Ito $\mathrm{H}$, Matsubara H. Refined balloon pulmonary angioplasty for inoperable patients with chronic thromboembolic pulmonary hypertension. Circ Cardiovasc Interv 2012;5:748-55.

15. Shimura N, Kataoka M, Inami $T$, Yanagisawa R, Ishiguro $H$, Kawakami T, et al. Additional percutaneous transluminal pul- 
monary angioplasty for residual or recurrent pulmonary hypertension after pulmonary endarterectomy. Int J Cardiol 2015;183:138-42.

16. Abrams D, Javidfar J, Farrand E, Mongero LB, Agerstrand CL,
Ryan P, et al. Early mobilization of patients receiving extracorporeal membrane oxygenation: a retrospective cohort study. Crit Care 2014;18:R38. 